\title{
Finite Distributive Concept Algebras
}

\author{
Bernhard Ganter • Léonard Kwuida
}

Received: 23 August 2005 / Accepted: 5 October 2006 /

Published online: 7 November 2006

(C) Springer Science + Business Media B.V. 2006

\begin{abstract}
Concept algebras are concept lattices enriched by a weak negation and a weak opposition. In Ganter and Kwuida (Contrib. Gen. Algebra, 14:63-72, 2004) we gave a contextual description of the lattice of weak negations on a finite lattice. In this contribution $^{1}$ we use this description to give a characterization of finite distributive concept algebras.
\end{abstract}

Key words formal concept analysis • negation • concept algebras • weakly dicomplemented lattices $\cdot$ superalgebraic lattices

Mathematics Subject Classifications (2000) $03 \mathrm{G} 25 \cdot 03 \mathrm{G} 10 \cdot 06 \mathrm{~B} 15$

\section{Motivation}

Weakly dicomplemented lattices have been introduced to capture the equational theory of concept algebras. These are bounded lattices equipped with two unary operations ${ }^{\Delta}$ and $\nabla$ called weak complementation and dual weak complementation, and satisfying for all $x$ and $y$ the following equations:
(1) $x^{\triangle \Delta} \leq x$
(1') $x^{\nabla \nabla} \geq x$
(2) $x \leq y \Longrightarrow x^{\Delta} \geq y^{\Delta}$,
(2') $x \leq \bar{y} \Longrightarrow x^{\nabla} \geq y^{\nabla}$,
(3) $(x \wedge y) \vee\left(x \wedge y^{\triangle}\right)=x$,
(3') $(x \vee y) \wedge(x \vee y \nabla)=x$.

${ }_{1}^{1}$ Major parts are taken from [5]
to concept lattices.

Dedicated to I. Rival.

B. Ganter

Institut für Algebra, TU Dresden, D-01062 Dresden, Germany

e-mail: Bernhard.Ganter@tu-dresden.de

L. Kwuida $(\bowtie)$

Mathematisches Institut, Universität Bern, CH-3012 Bern, Switzerland

e-mail: kwuida@math.unibe.ch 
The pair $\left(x^{\Delta}, x^{\nabla}\right)$ is called the weak dicomplement of $x$ and the pair $\left({ }^{\triangle}, \nabla\right)$ a weak dicomplementation. Concept algebras arose from the need to formalize the notion of "negation of a concept." These are concept lattices equipped with two unary operations ${ }^{\triangle}$ and $\nabla$ called weak negation and weak opposition, and defined for each formal concept $(A, B)$ by

$$
(A, B)^{\triangle}:=\left(\bar{A}^{\prime \prime}, \bar{A}^{\prime}\right) \quad \text { and } \quad(A, B)^{\nabla}:=\left(\bar{B}^{\prime}, \bar{B}^{\prime \prime}\right),
$$

where $\bar{X}$ stands for the set-complement of $X$ and ' the derivation in a formal context (see [6] for further details). Recall that a formal context is a triple $(G, M, I)$ of sets such that $I \subseteq G \times M$. The members of $G$ are called objects and those of $M$ attributes. If $(g, m) \in I$ the object $g$ is said to have $m$ as an attribute. For subsets $A \subseteq G$ and $B \subseteq M, A^{\prime}$ and $B^{\prime}$ are defined by

$$
\begin{aligned}
& A^{\prime}:=\{m \in M \mid \forall g \in A \quad g \operatorname{Im}\} \quad \text { and } \\
& B^{\prime}:=\{g \in G \mid \forall m \in B \quad g \operatorname{Im}\} .
\end{aligned}
$$

A formal concept of the context $(G, M, I)$ is a pair $(A, B)$ with $A \subseteq G$ and $B \subseteq M$ such that $A^{\prime}=B$ and $B^{\prime}=A$. In this case $A$ is called the extent and $B$ the intent of the concept $(A, B)$. The set of all formal concepts of the context $(G, M, I)$ is denoted by $\mathfrak{B}(G, M, I) \cdot \gamma g:=\left(\{g\}^{\prime \prime},\{g\}^{\prime}\right)$ and $\mu m:=\left(\{m\}^{\prime},\{m\}^{\prime \prime}\right)$ denote special concepts called respectively object concept and attribute concept. The hierarchy on concepts is captured by the inclusion relation on extents. In fact a concept $(A, B)$ is called a subconcept of a concept $(C, D)$ provided that $A \subseteq C$ (which is equivalent to $D \subseteq B$ ). We also call $(C, D)$ a superconcept of $(A, B)$ and write $(A, B) \leq(C, D)$. The poset $(\mathfrak{B}(G, M, I) ; \leq)$ is a complete lattice and is called the concept lattice of the context $(G, M, I)$ (see [4]). The concept algebra of a context $\mathbb{K}$ will be denoted by $\mathfrak{A}(\mathbb{K})$. i.e. $\mathfrak{A}(\mathbb{K}):=\left(\mathfrak{B}(\mathbb{K}), \wedge, \vee{ }^{\Delta}, \nabla, 0,1\right)$. The weak operations on $\mathfrak{A}(\mathbb{K})$ satisfy the equations (1) to $\left(3^{\prime}\right)$ above (see [6]). Concept algebras motivated the introduction of weakly dicomplemented lattices. Other examples are Boolean algebras (by duplicating the complementation), distributive double p-algebras, bounded lattices ( $x^{\Delta}=1$ for $x \neq$ 1 and $x^{\nabla}=0$ for $x \neq 0$, usually called the trivial weak dicomplementation), etc.... Thus the same bounded lattice might carry many weak dicomplementations.

On a lattice $L$ a weak dicomplementation $\left({ }^{\Delta_{1}}, \nabla_{1}\right)$ is said to be finer than a weak dicomplementation $\left({ }^{\Delta_{2}}, \nabla_{2}\right)$, written $\left({ }^{\Delta_{1}}, \nabla_{1}\right) \preceq\left({ }^{\Delta_{2}}, \nabla_{2}\right)$, if $x^{\Delta_{1}} \leq x^{\Delta_{2}}$ and $x^{\nabla_{1}} \geq x^{\nabla_{2}}$ for all $x$ in $L$. The "finer than" relation is an order relation on the set $\operatorname{Wd}(L)$ of all weak dicomplementations on a bounded lattice $L$. The poset $(\operatorname{Wd}(L), \preceq)$ admits a top element (the trivial weak dicomplementation). If the set $J(L)$ of all completely join-irreducible elements is $\bigvee$-dense and the set $M(L)$ of all completely meetirreducible elements is $\bigwedge$-dense ${ }^{2}$ then the weak dicomplementation of the concept algebra $\mathfrak{A}(J(L), M(L), \leq)$ (often mentioned as standard weak dicomplementation) is the bottom element of the poset $(\operatorname{Wd}(L), \preceq)$. In this case the poset of weak dicomplementations is a complete lattice. In fact for a nonempty family $\left\{\left(\triangle_{i}, \nabla^{i}\right) \mid i \in I\right\}$ of

\footnotetext{
${ }^{2}$ This is always the case for finite lattices. We often refer to this property as "lattices with enough irreducible elements." A special subclass is that of doubly founded lattices.
} 
weak dicomplementations on such a lattice $L$, the supremum is the operation $\left({ }^{\triangle_{I}}, \nabla_{I}\right)$ defined for all $x \in L$ by

$$
x^{\triangle_{I}}:=\bigvee\left\{x^{\triangle_{i}} \mid i \in I\right\} \quad \text { and } \quad x^{\nabla_{I}}:=\bigwedge\left\{x^{\nabla_{i}} \mid i \in I\right\} .
$$

Analogously the set $\mathrm{Wc}(L)$ of weak complementations on such an $L$ forms a complete lattice and contains the class of weak negations as a complete sublattice [5, Theorem 3.2.1]. Weak negations and weak oppositions behave independently (see Lemma 1 below). Thus the lattice of representable weak dicomplementations is a product of the lattice of weak negations and the lattice of weak oppositions. Since we are interested in representable weak dicomplementations, we can just concentrate on weak complementations.

Lemma 1 [3, Lemma 3] Let $L$ be a complete lattice and $\mathbb{K}:=(G, M, \leq)$ be a subcontext of $(L, L, \leq)$ such that $\mathfrak{B}(\mathbb{K}) \cong L$. For $g$ and $m$ in $L$ we set

$$
\mathbb{K}_{g}:=(G \cup\{g\}, M, \leq) \text { and } \mathbb{K}^{m}:=(G, M \cup\{m\}, \leq) .
$$

We have ${ }^{\Delta_{\mathbb{K}} m}={ }^{\Delta_{\mathbb{K}}}$ and ${\nabla \mathbb{K}^{m}}^{\nabla \mathbb{K}}$, as well as ${ }^{\nabla \mathbb{K}_{g}}={ }^{\nabla \mathbb{K}}$ and ${ }^{\Delta_{\mathbb{K}}} \geq^{\Delta_{\mathbb{K}}}$.

If a weakly dicomplemented lattice is (isomorphic to) a concept algebra of some context it is said to be representable ${ }^{3}$ (by this context). We will also speak about representable weak complementations. However not all complete lattices satisfying the equations (1) to $\left(3^{\prime}\right)$ are (isomorphic to) concept algebras. In this contribution we prove that finite distributive concept algebras are charaterized by the aforementioned equations. A start up example is the lattice product of a two-element and a $n$-element chain. Here all weak complementations are completely determined by the image of a single element, and are all weak negations (see Section 2). This section addresses the problem of determining weak dicomplementations. Section 3 is devoted to a contextual representation of the lattice of weak negations. Afterwards we examine in Section 4 the influence of the underlying lattice on the sublattice of representable weak complementations and then establish the characterization of finite distributive concept algebras.

\section{Determination of Weak Dicomplementations}

We start with a simple structure, a product of a two-element and an $n$-element chain.

\subsection{Weak Dicomplementations on the Lattice $\mathbf{2} \times \mathbf{n}$}

We want to determine all weak dicomplementations on the lattice $L:=\mathbf{2} \times \mathbf{n}$, the product of a two-element chain and an $n$-element chain. We use the labeling on

\footnotetext{
${ }^{3}$ In this contribution we use the term "representable" in the sense of the "strong representation problem" for weakly dicomplemented lattices (see [5, Section 1.4]).
} 
Figure 1 Weak

dicomplementations on $\mathbf{2} \times \mathbf{n}$.

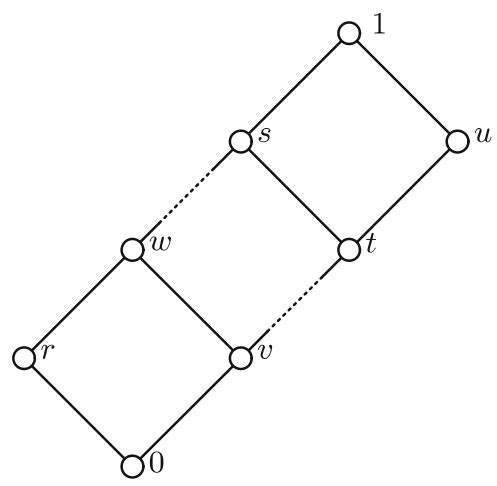

Figure 1. $L$ is a distributive lattice. The axioms of a weak complementation $f$ can be rewritten as follows:

(1) $f^{2} x \leq x$,

(2) $x \leq y \Longrightarrow f x \geq f y$ and

(3) $x \vee f x=1$.

Therefore $x \leq t$ implies $f x=1$ and $r \leq x \leq s$ implies $f x \geq u$, as well as $f u \geq r$. We set $A:=\{x \in[r, s] \mid f x=1\}$. If $A$ is empty then $f x=u$ for all $x \in[r, s]$. Since $f^{2} r \leq r$ holds we obtain the equality $f u=r$. We assume that $A$ is not empty and denote by $x_{1}$ the greatest element of $A$. For all $x$ in $[r, s]$, we have

$$
x \leq x_{1} \Longrightarrow f x=1 \text { and } x>x_{1} \Longrightarrow f x=u \text {. }
$$

We denote by $x_{2}$ the successor of $x_{1}$. Since $f^{2} x_{2} \leq x_{2}$ we obtain $f u \leq x_{2}$; if $f u<$ $x_{2}$ we would have $f^{2} u=1>u$, a contradiction. Then $f u=x_{2}$. The operation $f$ is completely determined by the image of $u$ in $[r, 1]$. There are exactly $n$ such operations (which are all weak complementations). Similarly there are also $n$ dual weak complementations $g$ on $L$, each determined by the image of $r$ in $[0, u]$. Thus $\mathrm{Wd}(L)$ is the product of two $n$ element chains (and contains exactly $n^{2}$ weak dicomplementations). They are all representable weak dicomplementations. In fact for a weak complementation $f$ and a dual weak complementation $g$ on $L$ the concept algebra of the context $(J(L) \cup\{f u\}, M(L) \cup\{g r\}, \leq)$ is (isomorphic to) the weakly dicomplemented lattice $(L, \wedge, \vee, f, g, 0,1)$ since

$$
\begin{aligned}
u^{\Delta} & \equiv\left(((J(L) \cup\{f u\}) \backslash \downarrow u)^{\prime \prime},((J(L) \cup\{f u\}) \backslash \downarrow u)^{\prime}\right) \\
& =(\downarrow f u \cap(J(L) \cup\{f u\}), \uparrow f u \cap(M(L) \cup\{g r\})) \\
& \equiv f u,
\end{aligned}
$$

where the relation $\equiv$ identifies a lattice element with its corresponding formal concept. Dually $r^{\nabla} \equiv g r$. 


\subsection{Determining Weak Dicomplementations}

We want to characterize weak dicomplementations on a given complete lattice $L$. Since clarifying ${ }^{4}$ a context does not alter the concept algebra structure, a representable weak dicomplementation on $L$ can always be represented by a pair $(G, M)$ of subsets of $L$ such that the concept algebra of the context $(G, M, \leq)$ is isomorphic to the given representable weakly dicomplemented lattice. To avoid confusion we sometimes index representable weak operations by their context name. This is usually the case if we deal with more than one context. A weak complementation ${ }^{\triangle}$ on $L$ is represented by a subset $G$ of $L$ iff $G$ is supremum dense and for all $x$ in $L$, $x^{\Delta}=\bigvee\{g \in G \mid g \not \leq x\}$. Dually, a dual weak complementation $\nabla$ on $L$ is represented by $M \subseteq L$ iff $M$ is infimum dense and for all $x$ in $L, x^{\nabla}=\bigwedge\{m \in M \mid m \nsucceq x\}$. The problem of finding a characterization of representable weak dicomplementations is still open. The idea is to first determine all weak complementations on L, and then check whether the lattice of weak complementations, via this determination, can be mapped to its sublattice of weak negations. The upcoming proposition gives an insight on which subsets can represent which weak dicomplementations. For a weak complementation ${ }^{\triangle}$, an element $u \in L$ is said to be ${ }^{\triangle}$-compatible if $u \leq x$ or $u \leq x^{\triangle}$ for all $x \in L$. Note that all $\vee$-irreducible elements are ${ }^{\triangle}$-compatible.

Proposition 1 If $G$ represents ${ }^{\triangle}$ on $L$ then $G \cup H$ also represents ${ }^{\triangle}$ if and only if all elements of $H$ are ${ }^{\Delta}$-compatible.

Proof $x^{\triangle}=\bigvee\{g \in G \mid g \not \leq x\} \leq \bigvee\{u \in G \cup H \mid u \not \leq x\}$. The inequality is proper iff there is some $u \in H$ with $u \not \leq x$ and $u \not \leq x^{\Delta}$. Thus $u$ must be $\Delta_{\text {-incompatible. }}$ Conversely if $u \in H$ is incompatible, then $u \not \leq x$ and $u \not \leq x^{\triangle}$ for some $x$, and for this $x$ the inequality is proper.

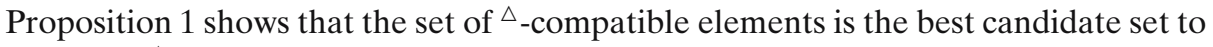
represent ${ }^{\triangle}$. A necessary condition is to be $\bigvee$-dense in $L$.

In general a weak dicomplementation on $L$ can be determined by its values on some subsets of $L$.

Lemma 2 [3] Let ${ }^{\triangle}$ be a weak complementation on $L$ and $M$ a $\bigwedge$-dense subset of $L$. For all $u \in L$ we have

(a) $\bigvee\left\{m^{\Delta} \mid m \geq u, m \in M\right\}=u^{\Delta}=\bigwedge\left\{n \mid n \geq m^{\Delta}\right.$ for all $\left.m \in M, m \geq u\right\}$.

(b) Weak complementations are determined by their values on any $\bigwedge$-dense subset.

(c) Weak complementations are determined by their $\Upsilon$-relation (on any $\wedge$-dense subset) defined by $m \Upsilon n: \Longleftrightarrow n \geq m^{\Delta}$.

For a context $(G, M, I)$ we define the relation $\perp$ on $M$ by

$$
m \perp n: \Longleftrightarrow m^{\prime} \cup n^{\prime}=G \text {. }
$$

\footnotetext{
${ }^{4} \mathrm{~A}$ context is clarified if for $g$ and $h$ (both objects or both attributes) $g^{\prime}=h^{\prime}$ holds only if $g=h$ holds. An object (resp. attribute) $g$ is reducible if there is a set $X$ of objects (resp. attributes) such that $g^{\prime}=X^{\prime}$ holds. A clarified context is reduced if all its objects and attributes are irreducible.
} 
Lemma 3 Let $L$ be a complete weakly complemented lattice such that a subset $G$ of its set of ${ }^{\triangle}$-compatible elements is $\bigvee$-dense. Let $M$ be a $\bigwedge$-dense subset of L. Then $\mathfrak{B}(G, M, \leq)$ is isomorphic to $L$ and the $\Upsilon$-relation of ${ }^{\Delta}$ is an order filter ${ }^{5}$ of the $\perp$ relation of $(G, M, \leq)$.

Proof Let ${ }^{\triangle}$ be a weak complementation on $L$ and $G$ a subset of the set of $\Delta_{-}$ compatible elements of $L$ that is $\bigvee$-dense. Let $M$ be a $\wedge$-dense subset of $L$; the concept lattice $\mathfrak{B}(G, M, \leq)$ is isomorphic to $L$ and for all $x \in L$ we have,

$$
x^{\triangle_{(G, M, \leq)}}=\bigvee\{g \in G \mid g \not \leq x\} \leq x^{\triangle} .
$$

Let $(m, n) \in \Upsilon$. From $m \Upsilon n$ we get

$$
m \geq n^{\triangle} \geq n^{\triangle_{(G, M, \leq)}} \quad \text { by }(\dagger) .
$$

For any element $g \in G$, if $g \notin n^{\prime}=\{h \in G \mid h \leq n\}$ then $g \not \leq n$. Thus $g \leq n^{\Delta} \leq m$ and $g \in m^{\prime}$. Thus $m \Upsilon n$ implies $m^{\prime} \cup n^{\prime}=G$, and so $m \perp n$.

$$
\text { i.e. }(m, n) \in \Upsilon \Longrightarrow(m, n) \in \perp \text {. }
$$

Now assume that $(m, n) \in \Upsilon$ and $(x, y) \geq(m, n)$. We have $m^{\Delta} \geq x^{\Delta}$ and $y \geq n \geq$ $m^{\Delta} \geq x^{\Delta}$; thus $y \geq x^{\Delta}$, and $x \Upsilon y$. This shows that $\Upsilon$ is an order filter of $\perp$.

Does the converse hold, i.e., do the $\Upsilon$-relations exhaust all order filters of $\perp$ ? A positive answer would imply the distributivity of the lattice of weak complementations (see Birkhoff's theorem Section 4).

Remark 1 In particular, if $L$ is a finite lattice, then its set of $\vee$-irreducible elements is a $\bigvee$-dense subset of ${ }^{{ }^{-}}$-compatible elements for any weak complementation on $L$. It is minimal. The $\Upsilon$-relation of any weak complementation on $L$ is an order filter of the $\perp$-relation of the reduced context of $L$. Having a smallest $\bigvee$-dense subset gives the opportunity to have all $\Upsilon$-relations as order filter of the same $\perp$-relation on any fixed $\bigwedge$-dense subset of $L$. Note that the smaller the set of objects is, the larger the $\perp$-relation is.

The relations $\Upsilon$ and $\perp$ are symmetric. In the rest of this contribution we adopt the following notations:

$$
\Gamma:=\{\{m, n\} \subseteq M \mid m \Upsilon n\} \text { and } T:=\{\{m, n\} \subseteq M \mid m \perp n\}
$$

\footnotetext{
${ }^{5}$ The $\Upsilon$-relation and the $\perp$-relation are both defined on $M$, and are subsets of $M \times M$ (ordered componentwise). 
Remark 2 If we consider the pair in $T$ to be of distinct elements, then $T$ can be empty. In this case, $T=\varnothing$ iff ${ }^{\triangle}$ is the trivial weak complementation. In fact,

$$
\begin{aligned}
T=\emptyset & \Longleftrightarrow \text { for all } m \in M, m^{\prime} \cup n^{\prime} \neq G \text { for all } n \in M \backslash\{m\} \\
& \Longleftrightarrow \text { for all } m \in M, G \backslash m^{\prime} \nsubseteq n^{\prime} \text { for all } n \in M \backslash\{m\} \\
& \Longleftrightarrow \text { for all } m \in M,\left(G \backslash m^{\prime}\right)^{\prime \prime} \nsubseteq n^{\prime} \text { for all } n \in M \backslash\{m\} \\
& \Longleftrightarrow \text { for all } m \in M, m^{\triangle} \not \leq n \text { for all } n \in M \backslash\{m\} \\
& \Longleftrightarrow \text { for all } m \in M, m^{\Delta}=1 .
\end{aligned}
$$

In the rest of this paper we assume that $T$ is nonempty.

\section{Lattice of Representable Weak Complementations}

We denote by $\operatorname{Ext}(\mathbb{K})$ the lattice of all extents of the context $\mathbb{K}:=(G, M, I)$. The context $\mathbb{R}:=(\operatorname{Ext}(\mathbb{K}), T, \mathcal{R})$ is defined by:

$$
U \mathcal{R}\{m, n\}: \Longleftrightarrow U \subseteq m^{\prime} \text { or } U \subseteq n^{\prime} .
$$

$(-)^{\mathcal{R}}$ denotes the derivation operation in $\mathbb{R}$. We set $(-)^{c}:=(-)^{\mathcal{R} \mathcal{R}}$. For $U$ in $\operatorname{Ext}(\mathbb{K})$ we have

$$
\{U\}^{c}=\{W \in \operatorname{Ext}(\mathbb{K}) \mid U \mathcal{R}\{m, n\} \Longrightarrow W \mathcal{R}\{m, n\} \text { for all }\{m, n\} \in T\} .
$$

The relation $I$ of $\mathbb{K}$ is extended by $I_{e}$ on $\left(G \uplus \operatorname{Ext}(\mathbb{K}), M, I_{e}\right)$ as follows:

$$
I_{e} \cap G \times M=I \quad \text { and } \quad U I_{e} m: \Longleftrightarrow U \subseteq m^{\prime} \quad \text { for } U \in \operatorname{Ext}(\mathbb{K}) .
$$

$I_{e}$ will also denote its restriction on $\left(G \uplus \mathcal{H}, M, I_{e}\right)$ with $\mathcal{H} \subseteq \operatorname{Ext}(\mathbb{K})$. These contexts have isomorphic concept lattices and define the same weak opposition. However the weak negations they define can be different (see Lemma 1). As above $(-)^{I_{e}}$ denotes the derivation in the extended contexts. Recall that an extent $U$ is called ${ }^{\triangle}$-compatible if and only if $U \subseteq A$ or $U \subseteq \bar{A}^{\prime \prime}$ for all extents $A$, and that the weak negation of an extented context coincides with the old weak negation if and only if all new objects are ${ }^{\Delta}$-compatible in the old context. Here is a characterization of the compatibility by means of the $\perp$-relation.

Lemma 4 For $U \in \operatorname{Ext}(\mathbb{K})$

$$
U \in T^{\mathcal{R}} \Longleftrightarrow U \text { is }{ }^{\Delta_{\text {-compatible in }}} \mathfrak{A}(\mathbb{K}) \Longleftrightarrow{ }^{\Delta_{(G, M, I)}}=^{\triangle_{\left.(G \uplus U U\}, M, I_{e}\right)}}
$$

Proof The second equivalence follows from Proposiotion 1. The first equivalence is obtained by contraposition: suppose $U \notin T^{\mathcal{R}}$; then there is $\{m, n\} \in T$ such that $U \nsubseteq$ $m^{\prime}$ and $U \nsubseteq n^{\prime}$; for $A:=m^{\prime}$ we have $U \nsubseteq A$ and $U \nsubseteq n^{\prime} \supseteq \bar{A}^{\prime \prime}$. Conversely suppose that $U$ is $\triangle^{\triangle}$-incompatible in $\mathfrak{B}(\mathbb{K})$; then there is $A \in \operatorname{Ext}(\mathbb{K})$ such that $U \nsubseteq A$ and $U \nsubseteq \bar{A}^{\prime \prime}$. There exist $m \in A^{\prime}$ with $U \nsubseteq m^{\prime}$, and $n \in \bar{A}^{\prime}$ with $U \nsubseteq n^{\prime}$ such that $m^{\prime} \cup n^{\prime} \supseteq$ $A \cup \bar{A}=G$. 
Lemma 5 For $\mathcal{H} \subseteq \operatorname{Ext}(\mathbb{K}), \mathcal{H}^{c}$ is the set of $\Delta_{\left(G \uplus \mathcal{H}, M, I_{e}\right)}$-compatible elements. Hence, $\Delta_{\left(G \uplus \mathcal{H}, M, I_{e}\right)}={ }^{\left.\Delta_{(G \uplus} \mathcal{H} C, M, I_{e}\right)}$.

Proof

$$
\begin{aligned}
W \in \mathcal{H}^{c} \Longleftrightarrow & (\{m, n\} \in T \text { and } U \mathcal{R}\{m, n\} \text { for all } U \in \mathcal{H} \Longrightarrow W \mathcal{R}\{m, n\}) \\
\Longleftrightarrow & \left(m^{\prime} \cup n^{\prime}=G \text { and }\left(U \subseteq m^{\prime} \text { or } U \subseteq n^{\prime}\right) \forall U \in \mathcal{H} \Longrightarrow W \subseteq m^{\prime}\right. \\
& \text { or } \left.W \subseteq n^{\prime}\right) \\
\Longleftrightarrow & \left(m^{I_{e}} \cup n^{I_{e}}=G \uplus \mathcal{H} \Longrightarrow W \subseteq m^{\prime} \text { or } W \subseteq n^{\prime}\right) \\
\Longleftrightarrow & W \text { is } \triangle_{\left(G \uplus \mathcal{H}, M, I_{e}\right)} \text {-compatible. }
\end{aligned}
$$

Lemma 6 Let L be a lattice with two weak complementations ${ }^{\Delta_{1}}$ and ${ }^{\Delta_{2}}$. If ${ }^{\Delta_{2}} \leq^{\Delta_{1}}$ then every ${ }^{\Delta_{1}}$-compatible element is also ${ }^{\Delta_{2}}$-compatible.

Proof Let $u$ be a ${ }^{\Delta_{1}}$-compatible element. For all $x \in L, u \leq x$ or $u \leq x^{\Delta_{1}}$. This implies $u \leq x$ or $u \leq x^{\Delta_{2}}$ since $x^{\triangle_{1}} \leq x^{\Delta_{2}}$. Thus $u$ is ${ }^{\Delta_{2}}$-compatible.

From this, we can now prove the following result

Theorem 1 [Contextual description of the lattice of weak negations] Let $\mathbb{K}$ be $a$ reduced ${ }^{6}$ context. The concept lattice of the context $(\operatorname{Ext}(\mathbb{K}), T, \mathcal{R})$ is isomorphic to the lattice of representable weak complementations on $\mathfrak{B}(\mathbb{K})$.

Proof We denote by $\mathrm{Wn}(L)$ the set of representable weak complementations on a lattice $L$. By Lemma 1 and Lemma 5 the assignment

$$
\psi:\left(\mathcal{H}, \mathcal{H}^{\mathcal{R}}\right) \mapsto^{\left.{ }_{(G \uplus \mathcal{H}}, M, I_{e}\right)}
$$

defines an increasing map from $\mathfrak{B}(\operatorname{Ext}(\mathbb{K}), T, \mathcal{R})$ to $\mathrm{Wn}(\mathfrak{B}(\mathbb{K}))$. Let $\mathcal{H}_{1}$ and $\mathcal{H}_{2}$ be subsets of $\operatorname{Ext}(\mathbb{K})$. We assume that

$$
\left.\Delta_{(G \uplus \mathcal{H}}, M, I_{e}\right)={ }^{\left.\Delta_{(G \uplus \mathcal{H}}, M, I_{e}\right)} ; \quad \text { then }{ }^{\Delta_{1}}:={ }^{\left.\Delta_{(G \uplus \mathcal{H}}, M, I_{e}\right)}={ }^{\left.\Delta_{(G \uplus \mathcal{H}}, M, M, I_{e}\right)}=:{ }^{\Delta_{2}} .
$$

As ${ }^{\Delta_{1}}={ }^{\Delta_{2}}$ we have (by Lemma 5) for each $W \in \operatorname{Ext}(\mathbb{K})$,

$$
W \in \mathcal{H}_{1}^{c} \Longleftrightarrow W \text { is }{ }^{\Delta_{1}} \text {-compatible } \Longleftrightarrow W \text { is }{ }^{\Delta_{2}} \text {-compatible } \Longleftrightarrow W \in \mathcal{H}_{2}^{c} \text {. }
$$

Thus the map

$$
\phi:{ }_{\left(G \uplus \mathcal{H}, M, I_{e}\right)} \mapsto\left(\mathcal{H}^{c}, \mathcal{H}^{\mathcal{R}}\right)
$$

is well defined from $\operatorname{Wn}(\mathfrak{B}(\mathbb{K}))$ to $\mathfrak{B}(\operatorname{Ext}(\mathbb{K}), T, \mathcal{R})$. The compositions $\psi \circ \phi$ and $\phi \circ \psi$ are identity maps. In fact,

$$
\phi \circ \psi\left(\mathcal{H}, \mathcal{H}^{\mathcal{R}}\right)=\phi\left(^{\triangle_{\left(G \uplus \mathcal{H}, M, I_{e}\right)}}\right)=\left(\mathcal{H}^{c}, \mathcal{H}^{\mathcal{R}}\right)=\left(\mathcal{H}, \mathcal{H}^{\mathcal{R}}\right) \quad \text { for all }\left(\mathcal{H}, \mathcal{H}^{\mathcal{R}}\right) \in \mathfrak{B}(\mathbb{R}),
$$

\footnotetext{
${ }^{6}$ See Remark 1.

悬 Springer
} 
Figure 2 Free distributive lattice generated by three elements and the corresponding reduced context.

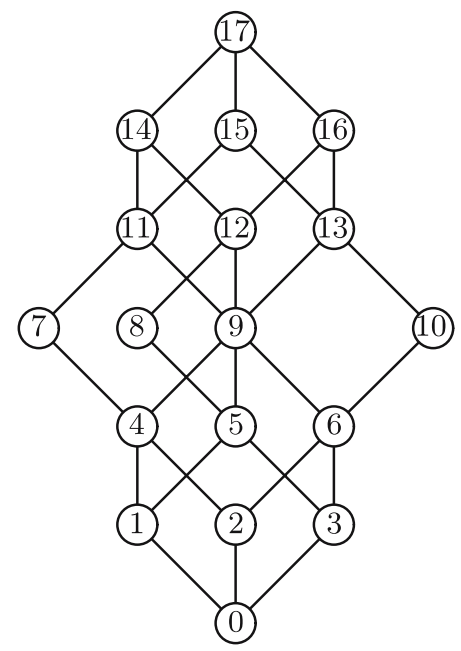

and for all $\mathcal{H} \subseteq \operatorname{Ext}(\mathbb{K})$ we have

$$
\psi \circ \phi\left({ }^{\triangle_{\left(G \uplus \mathcal{H}, M, I_{e}\right)}}\right)=\psi\left(\mathcal{H}^{c}, \mathcal{H}^{\mathcal{R}}\right)={ }^{\left.\triangle_{(G \uplus \mathcal{H}}, M, I_{e}\right)}={ }^{\triangle_{\left(G \uplus \mathcal{H}, M, I_{e}\right)}} \quad \text { by Lemma } 5 .
$$

Thus $\psi$ and $\phi$ are bijections and inverse to each other. To achieve the proof it remains to show that $\phi$ is also increasing. To see this assume that

$$
\Delta_{1}:=^{\left.\Delta_{(G \uplus \mathcal{H}}, M, I_{e}\right)} \leq \triangle_{\left(G \uplus \mathcal{H}_{2}, M, I_{e}\right)}=:^{\triangle_{2}} .
$$

By Lemma 6 the set $\mathcal{H}_{2}^{c}$ of ${ }^{\Delta_{2}}$-compatible extents contains the set of ${ }^{\Delta_{1}}$-compatible extents $\mathcal{H}_{1}^{c}$. Therefore ${ }_{\left(G \uplus \mathcal{H}_{1}, M, I_{e}\right)} \leq \Delta_{\left(G \uplus \mathcal{H}_{2}, M, I_{e}\right)}$ implies $\mathcal{H}_{1}^{c} \subseteq \mathcal{H}_{2}^{c}$ and $\phi$ is increasing. Thus $\phi$ and $\psi$ are order preserving bijections, inverse each other and are then lattice isomorphisms.

Before we proceed further let us have a look at an example.

Example 1 We consider the free distributive lattice generated by three elements. Its reduced context has the attribute set $M:=\{7,8,10,14,15,16\}$ and the object set $J:=\{1,2,3,7,8,10\}$ (see Figure 2).

The set of orthogonal pairs of attributes is given by

$$
T=\{\{14,15\},\{14,16\},\{14,10\},\{15,16\},\{15,8\},\{16,7\}\} .
$$

The context $\mathbb{R}$ is given on Figure 3 . All objects from 0 to 10 , and the object 17 are reducible. The resulting context is a copy of the context on Figure 2. Thus the lattice of concrete weak complementations on the free lattice generated by three elements is isomorphic to this lattice.

In the next section we show that some properties of the initial lattice can be carried over to the lattice of representable weak complementations. 


\section{Distributive Lattices}

We first recall some results needed in this section. Completely distributive complete lattices in which the set of (completely) $\bigvee$-irreducible elements is $\bigvee$-dense are called superalgebraic, being characterized by the property that every element is a join of supercompact (i.e. $\bigvee$-prime) elements. $J(L)$ denotes the set of supercompact elements of $L$.

Birkhoff's theorem. If a lattice $L$ is superalgebraic then $x \mapsto \downarrow x \cap J(L)$ describes an isomorphism of $L$ onto the closure system of all order ideals of $(J(L), \leq)$. Conversely for every ordered set $(P, \leq)$ the closure system of all order ideals of $(P, \leq)$ is superalgebraic.

Contraordinal scale. For an arbitrary ordered set $(P, \leq)$ the context $(P, P, \ngtr)$ (called contraordinal scale) is reduced. Its concept lattice is a copy of the lattice of order ideals of $(P, \leq)$.

Contranominal scale. For a set $S$ the context $(S, S, \neq)$ (contranominal scale) is reduced. Its concept lattice is isomorphic to the power set lattice of $S$.

Let $L$ be a superalgebraic lattice. There is a poset $(P, \leq)$ such that $\mathfrak{B}(P, P, \nsupseteq)$ is isomorphic to $L$. The relation $\perp$ on $P$ is characterized by

$$
m \perp n \Longleftrightarrow \overline{\uparrow m} \cup \uparrow n=P \Longleftrightarrow \uparrow m \cap \uparrow n=\emptyset .
$$

Lemma 7 The relation $\leq$ defined on $T=\{\{m, n\} \subseteq P \mid m \perp n\}$ by

$$
\{x, y\} \leq\{s, t\}: \Longleftrightarrow\{x, y\} \subseteq \downarrow\{s, t\}
$$

Figure 3 Context of all weak negations on the free distributive lattice generated by three elements.

\begin{tabular}{|c||c|c|c|c|c|c|}
\hline & $\{14,15\}$ & $\{14,16\}$ & $\{14,10\}$ & $\{15,16\}$ & $\{15,8\}$ & $\{16,7\}$ \\
\hline \hline 0 & $\times$ & $\times$ & $\times$ & $\times$ & $\times$ & $\times$ \\
\hline 1 & $\times$ & $\times$ & $\times$ & $\times$ & $\times$ & $\times$ \\
\hline 2 & $\times$ & $\times$ & $\times$ & $\times$ & $\times$ & $\times$ \\
\hline 3 & $\times$ & $\times$ & $\times$ & $\times$ & $\times$ & $\times$ \\
\hline 4 & $\times$ & $\times$ & $\times$ & $\times$ & $\times$ & $\times$ \\
\hline 5 & $\times$ & $\times$ & $\times$ & $\times$ & $\times$ & $\times$ \\
\hline 6 & $\times$ & $\times$ & $\times$ & $\times$ & $\times$ & $\times$ \\
\hline 7 & $\times$ & $\times$ & $\times$ & $\times$ & $\times$ & $\times$ \\
\hline 8 & $\times$ & $\times$ & $\times$ & $\times$ & $\times$ & $\times$ \\
\hline 9 & $\times$ & $\times$ & $\times$ & $\times$ & $\times$ & $\times$ \\
\hline 10 & $\times$ & $\times$ & $\times$ & $\times$ & $\times$ & $\times$ \\
\hline 11 & $\times$ & $\times$ & $\times$ & $\times$ & $\times$ & \\
\hline 12 & $\times$ & $\times$ & $\times$ & $\times$ & & $\times$ \\
\hline 13 & $\times$ & $\times$ & & $\times$ & $\times$ & $\times$ \\
\hline 14 & $\times$ & $\times$ & $\times$ & & & \\
\hline 15 & $\times$ & & & $\times$ & $\times$ & \\
\hline 16 & & $\times$ & & $\times$ & & $\times$ \\
\hline 17 & & & & & & \\
\hline
\end{tabular}


is an order relation.

Proof Reflexivity and transitivity are obvious. To prove antisymmetry, we assume $\{x, y\} \leq\{s, t\}$ and $\{s, t\} \leq\{x, y\}$. Note that $x$ and $y$ cannot together be less than $s$ or than $t$; otherwise $s$ or $t$ would belong to $\uparrow x \cap \uparrow y$ which is empty. Even the assertion " $x \leq s, \quad y \leq t$ and $s \leq y, t \leq x$ " cannot hold; otherwise we would have $x \leq s \leq y \leq$ $t \leq x$ which is a contradiction. Without loss of generality our assumption implies $x \leq$ $s, y \leq t$ and $s \leq x, t \leq y$; therefore $\{x, y\}=\{s, t\}$ and $\leq$ is antisymmetric. Thus $\leq$ is an order relation on $T$.

Lemma 8 For any poset $(P, \leq)$ the intents of $(\operatorname{Ext}(P, P, \ngtr), T, \mathcal{R})$ are exactly the order filters of $(T, \leq)$.

Proof Let $U \in \operatorname{Ext}(P, P, \ngtr)$ with $\{m, n\} \in U^{\mathcal{R}}$ and $\{x, y\} \geq\{m, n\}$. On one hand $\{m, n\} \in U^{\mathcal{R}}$ if and only if $U \subseteq m^{\prime}$ or $U \subseteq n^{\prime}$; up to permuting $m$ and $n$ we have

$$
\{x, y\} \geq\{m, n\} \Longleftrightarrow x \geq m \text { and } y \geq n \Longleftrightarrow \overline{\uparrow x} \supseteq \overline{\uparrow m} \text { and } \overline{\uparrow y} \supseteq \overline{\uparrow n} \text {. }
$$

Hence, $\{x, y\} \geq\{m, n\}$ implies $x^{\prime} \supseteq m^{\prime} \supseteq U$ or $y^{\prime} \supseteq n^{\prime} \supseteq U$, and by then $\{x, y\} \in U^{\mathcal{R}}$. Thus $U^{\mathcal{R}}$ is an order filter. For any intent $B$ of $(\operatorname{Ext}(P, P, \ngtr), T, \mathcal{R})$, there is $\mathcal{U} \subseteq$ $\operatorname{Ext}(P, P, \ngtr)$ such that $B=\mathcal{U}^{\mathcal{R}}=\bigcap\left\{U^{\mathcal{R}} \mid U \in \mathcal{U}\right\}$, and is an order filter. Thus all intents of $(\operatorname{Ext}(P, P, \ngtr), T, \mathcal{R})$ are order filter of $(T, \leq)$.

Conversely, let $B$ be an order filter of $(T, \leq)$. We want to prove that $B=B^{\mathcal{R} \mathcal{R}}$. Let $\{m, n\} \notin B$. We have

$$
\begin{aligned}
\{m, n\} \notin B & \Longleftrightarrow \forall\{s, t\} \in B,\{s, t\} \not \leq\{m, n\} \\
& \Longleftrightarrow \forall\{s, t\} \in B, m \ngtr s \text { and } n \nsupseteq s \text { or } m \ngtr t \text { and } n \ngtr t \\
& \Longleftrightarrow \forall\{s, t\} \in B, \downarrow\{m, n\} \subseteq s^{\ngtr} \text { and } \downarrow\{m, n\} \subseteq t^{\ngtr} \\
& \Longleftrightarrow \forall\{s, t\} \in B, \downarrow\{m, n\} \mathcal{R}\{s, t\} \\
& \Longleftrightarrow \downarrow\{m, n\} \in B^{\mathcal{R}} .
\end{aligned}
$$

Moreover, $\downarrow\{m, n\} \nsubseteq m^{\ngtr}$ and $\downarrow\{m, n\} \nsubseteq \subseteq n^{\ngtr}$ imply $\operatorname{not}(\downarrow\{m, n\} \mathcal{R}\{m, n\})$. Thus $\{m, n\} \notin B^{\mathcal{R} \mathcal{R}}$. Hence, $B=B^{\mathcal{R} \mathcal{R}}$.

We denote by $\mathrm{oF}(T, \leq)$ (resp. oI $(T, \leq))$ the superalgebraic lattice of order filters (resp. ideals) of $(T, \leq)$, and write $\cong$ d to mean "is dual isomorphic to". Int $(\mathbb{K})$ denotes the lattice of intents of a context $\mathbb{K}$. Note that $\operatorname{Ext}(\mathbb{K}) \cong{ }^{d} \operatorname{Int}(\mathbb{K})$.

Theorem 2 The lattice of representable weak complementations on any superalgebraic lattice is superalgebraic.

Proof Let $L$ be a superalgebraic lattice. There is a poset $(P, \leq)$ (of supercompact elements of $L)$ such that $\mathfrak{B}(P, P, \ngtr) \cong L$. By Theorem 1 the lattice of representable weak complementations on $L$ is isomorphic to $\mathfrak{B}(\operatorname{Ext}(P, P, \ngtr), T, \mathcal{R})$. Using Lemma 8 we get

$$
\mathfrak{B}(\operatorname{Ext}(P, P, \nsupseteq), T, \mathcal{R}) \cong^{d} \operatorname{Int}(\operatorname{Ext}(P, P, \nsupseteq), T, \mathcal{R})=\mathrm{oF}(T, \leq) \cong{ }^{d} \mathrm{oI}(T, \leq),
$$


which is superalgebraic (by Birkhoff's theorem).

Corollary 1 The lattice of representable weak complementations on a complete atomic Boolean algebra (resp. a finite distributive lattice) is a complete atomic Boolean algebra (resp. a finite distributive lattice).

Proof Note that finite distributive lattices and complete atomic Boolean algebras are special cases of superalgebraic lattices. In the second case, the context $(P, P, \nsupseteq)$ is exactly $(P, P, \neq)$, and the lattice of representable weak complementations is a copy of the lattice of order filter of the poset $(T,=)$, which is a complete atomic Boolean algebra.

We are now ready to give the main results of this contribution.

Theorem 3 For any superalgebraic lattice L, the weak complementations on L form a superalgebraic lattice isomorphic to the lattice of all order ideals of

$$
T=\{\{m, n\} \subseteq P \mid \uparrow m \cap \uparrow n=\emptyset\},
$$

where $P$ is the set of all $\bigvee$-prime elements of $L$ and $T$ is ordered by

$$
\{x, y\} \leq\{m, n\} \Longleftrightarrow\{x, y\} \subseteq \downarrow\{m, n\} .
$$

Proof Let ${ }^{\triangle}$ be a weak complementation on a superalgebraic lattice $L$. By Lemma 2, $\triangle$ is completely determined by its $\Upsilon$-relation on $P$. This $\Upsilon$-relation is, by Lemma 3 , an order filter of the $\perp$-relation on $P$. The $\Upsilon$-relation and $\perp$-relation are symmetric and are exactly determined by their respective factorization (with respect to the symmetry)

$$
\Gamma=\{\{m, n\} \mid m, n \in M, m \Upsilon n\} \quad \text { and } \quad T=\{\{m, n\} \mid m, n \in M, m \perp n\} .
$$

The factorisation of the order $\leq$ on $\perp$ corresponds to the order $\leq$ on $T$ (see Lemma 7) and turns $\Gamma$ into an order filter of the poset $(T, \leq)$. The assignment $\eta:{ }^{\Delta} \mapsto \eta\left({ }^{\triangle}\right):=$ $T \backslash \Gamma$ defines a map from $\operatorname{Wc}(L)$ to oI $(T, \leq)$. By Lemma 2 we have

$$
\Delta_{1} \leq^{\Delta_{2}} \Longleftrightarrow \Upsilon_{1} \supseteq \Upsilon_{2} \Longleftrightarrow \Gamma_{1} \supseteq \Gamma_{2} \Longleftrightarrow T \backslash \Gamma_{1} \subseteq T \backslash \Gamma_{2} \Longleftrightarrow \eta\left(\left(^{\Delta_{1}}\right) \leq \eta\left(\left(^{\Delta_{2}}\right) .\right.\right.
$$

Hence, $\eta$ is an order embedding of $\mathrm{Wc}(L)$ into oI $(T, \leq)$.

Conversely if $J$ is an order ideal of $(T, \leq)$, then

$$
T \backslash J \in \mathrm{oF}(T, \leq)=\operatorname{Int}(\operatorname{Ext}(P, P, \ngtr), T, \mathcal{R}) \quad \text { by Lemma } 8 .
$$

Thus $\left((T \backslash J)^{\mathcal{R}},(T \backslash J)\right) \in \mathfrak{B}(\mathbb{R})$ and $\psi\left((T \backslash J)^{\mathcal{R}},(T \backslash J)\right) \in \mathrm{Wn}(L) \subseteq \mathrm{Wc}(L)$. Thus $\zeta: J \mapsto \psi\left((T \backslash J)^{\mathcal{R}},(T \backslash J)\right)$ defines a map from oI $(T, \leq)$ to $\operatorname{Wc}(L)$. Moreover, $\eta \circ$ $\zeta(J)=J$. In fact, setting $\mathcal{H}=(T \backslash J)^{\mathcal{R}}$ we have

$$
\zeta(J)=\psi\left(\mathcal{H}, \mathcal{H}^{\mathcal{R}}\right)=^{\Delta_{(P \uplus \mathcal{H}, P, \nsupseteq e)}} \quad \text { and } \quad \eta \circ \zeta(J)=T \backslash \Gamma_{\zeta(J)}=T \backslash \Gamma_{\Delta_{(P \uplus \mathcal{H}, P, \nsucceq e)}} .
$$

Thus $\eta \circ \zeta(J)=J$ iff $T \backslash \Gamma_{\Delta_{(P \uplus \mathcal{H}, P, \ngtr e)}}=J$ iff $\Gamma_{\Delta_{(P \uplus \mathcal{H}, P, \ngtr e)}}=T \backslash J$. Thus we should prove that $\{m, n\} \notin J$ iff $\mu m \geq \mu n^{\Delta_{\left(P_{\uplus} \mathcal{H}, P_{,} \Varangle_{e}\right)}}$ for all $\{m, n\} \in T$. Note that

$$
\{m, n\} \notin J \Longleftrightarrow\{m, n\} \ngtr\{a, b\} \quad \forall\{a, b\} \in J \Longleftrightarrow\{m, n\} \in J \ngtr .
$$


Thus $T \backslash J=J \nsupseteq$ and $\mathcal{H}=J \nsupseteq \mathcal{R}$.

$$
\begin{aligned}
& \Gamma_{\triangle_{\left(P \uplus \mathcal{H}, P, \geq_{e}\right)}}=\left\{\{m, n\} \in T \mid \mu m \geq \mu n^{\Delta_{\left(P_{\uplus} \mathcal{H}, P, \ngtr e\right)}}\right\}
\end{aligned}
$$

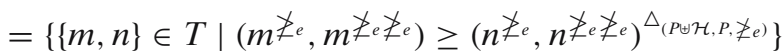

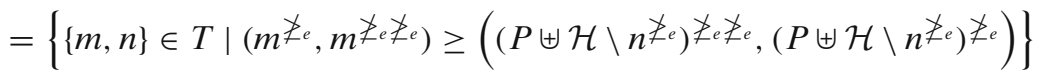

$$
\begin{aligned}
& =\left\{\{m, n\} \in T \mid m^{\ngtr_{e}} \supseteq\left(P \uplus \mathcal{H} \backslash n \chi_{e}\right) \nsucceq_{e} \Varangle_{e}\right\} \\
& =\left\{\{m, n\} \in T \mid m \ngtr_{e} \supseteq P \uplus \mathcal{H} \backslash n \nsupseteq e\right\} \\
& =\left\{\{m, n\} \in T \mid x \notin n^{\ngtr e} \text { implies } x \in m^{\ngtr e} \text { for all } x \in P \uplus \mathcal{H}\right\} \\
& =\left\{\{m, n\} \in T \mid \operatorname{not}\left(x \Varangle_{e} n\right) \text { implies } x \Varangle_{e} m \text { for all } x \in P \uplus \mathcal{H}\right\} \\
& =\{\{m, n\} \in T \mid(\operatorname{not}(x \nsupseteq n) \text { implies } x \ngtr m \text { for all } x \in P) \text { and } \\
& \left.\left(\operatorname{not}\left(U \Varangle_{e} n\right) \text { implies } U \Varangle_{e} m \text { for all } U \in \mathcal{H}\right)\right\} \\
& =\left\{\{m, n\} \in T \mid\left(\operatorname{not}\left(x \in n^{\ngtr}\right) \text { implies } x \in m^{\ngtr} \text { for all } x \in P\right)\right. \text { and } \\
& \left(\operatorname{not}\left(U \subseteq n^{\ngtr}\right) \text { implies } U \subseteq m^{\ngtr} \text { for all } U \in \mathcal{H}\right) \text { \} } \\
& =\left\{\{m, n\} \in T \mid\left(x \notin n^{\ngtr} \text { implies } x \in m^{\ngtr} \text { for all } x \in P\right)\right. \text { and } \\
& \left.\left(U \subseteq n^{\ngtr} \text { or } U \subseteq m^{\ngtr} \text { for all } U \in \mathcal{H}\right)\right\} \\
& =\{\{m, n\} \in T \mid U \mathcal{R}\{m, n\} \text { for all } U \in \mathcal{H}\}, \quad \text { since } m \ngtr \cup n^{\ngtr}=P \\
& =\{\{m, n\} \in T \mid U \mathcal{R}\{m, n\} \text { for all } U \in J \ngtr \mathcal{R}\} \\
& =J \ngtr \mathcal{R} \mathcal{R}=(T \backslash J)^{\mathcal{R} \mathcal{R}}=T \backslash J, \quad \text { since } T \backslash J \in \operatorname{Int}(\mathbb{R}) .
\end{aligned}
$$

Thus $\eta$ is an isomorphism of $\mathrm{Wc}(L)$ onto $\mathrm{oI}(T, \leq)$.

\section{Theorem 4}

(a) The lattice $\mathrm{Wc}(L)$ of weak complementations on a superalgebraic lattice $L$ is isomorphic to its sublattice $\mathrm{Wn}(L)$ of representable weak complementations.

(b) On finite distributive lattices all weak complementations are representable. i.e. each weak complementation on a finite distributive lattice is a weak negation.

(c) All finite distributive weakly dicomplemented lattices are (isomorphic to) concept algebras.

Proof (a) follows from

$$
\mathrm{Wc}(L) \cong \mathrm{oI}(T, \leq) \cong{ }^{d} \mathrm{oF}(T, \leq)=\operatorname{Int}(\mathbb{R}) \cong{ }^{d} \mathfrak{B}(\mathbb{R}) \cong \mathrm{Wn}(L),
$$

where $\mathbb{R}$ is the context $(\operatorname{Ext}(P, P, \ngtr), T, \mathcal{R})$ and $\operatorname{Int}(\mathbb{R})$ the lattice of its intents.

In the finite case $\operatorname{Wc}(L)$ is finite and has the same cardinality as its subset $\mathrm{Wn}(L)$. Thus $\mathrm{Wn}(L)=\mathrm{Wc}(L)$ and (b) is proved. i.e. All weak complementations are weak negations. Dually we obtain that each dual weak complementation is a weak opposition and (c) is proved (see Lemma 1 and Proposition 1). 


\section{Conclusion}

The main result of the present contribution states that finite distributive concept algebras are exactly finite distributive lattices with two unary operations ${ }^{\Delta}$ and $\nabla$ satisfying the following equations for all $x$ and $y$ :
(1) $x^{\Delta \Delta} \leq x$,
(1') $\quad x \nabla \nabla \geq x$
(2) $x \leq y \Longrightarrow x^{\Delta} \geq y^{\Delta}$,
(2') $x \leq \bar{y} \Longrightarrow x^{\nabla} \geq y^{\nabla}$
(3) $(x \wedge y) \vee\left(x \wedge y^{\triangle}\right)=x$,
(3') $(x \vee y) \wedge\left(x \vee y^{\nabla}\right)=x$.

In this case the condition (3) is equivalent to $y \vee y^{\triangle}=1$ and the condition ( $\left.3^{\prime}\right)$ is equivalent to $y \wedge y \nabla=0$. Now that we have a characterization of finite distributive concept algebras the next step would be to consider standard problems such as free structures, decomposition, etc.... Congruences of finite distributive concept algebras have been described [2]. Although $\mathrm{Wn}(L)$ and $\mathrm{Wc}(L)$ are isomorphic for a superalgebraic lattice $L$, it is still not clear whether they are equal.

\section{References}

1. Davey, B.A., Priestley, H.A.: Introduction to Lattices and Order. Cambridge University Press, New York, Second Edition (2002)

2. Ganter, B.: Congruence of finite distributive concept algebras. In: Lecture Notes in Computer Science, vol. 2961, pp. 128-141. Springer, Berlin Heidelberg New York (2004)

3. Ganter, B., Kwuida, L.: Representable weak dicomplementations on finite lattices. Contrib. Gen. Algebra 14, 63-72 (2004) (J. Heyn Klagenfurt)

4. Ganter, B., Wille, R.: Formal Concept Analysis: Mathematical Foundations. Springer, Berlin Heidelberg New York (1999)

5. Kwuida, L.: Dicomplemented lattices. A contextual generalization of Boolean algebras. Shaker Verlag, Aachen (2004)

6. Wille, R.: Boolean concept logic. In: Lecture Notes in Computer Scince, vol. 1867, pp. 317-331. Springer, Berlin Heidelberg New York (2000) 